\title{
Topological Hermitian cobordism
}

\author{
Po Hu • Igor Kriz
}

Received: 27 July 2013 / Accepted: 6 December 2014 / Published online: 8 January 2015

(C) Tbilisi Centre for Mathematical Sciences 2015

\begin{abstract}
Extending our method for investigating Real cobordism (which was recently used by Hill, Hopkins and Ravenel in their solution of the Kervaire invariant 1 problem), we investigate the $R O(G)$-graded homotopy groups of a (non-complete) $\mathbb{Z} / 2 \times \mathbb{Z} / 2$-equivariant spectrum called topological Hermitian cobordism. The methods of this paper may be useful in computing the homotopy groups of other $G$-equivariant spectra where $G \neq \mathbb{Z} / 2$.
\end{abstract}

Keywords Cobordism · Hermitian K-theory · Equivariant stable homotopy theory

Mathematics Subject Classification 57R85 - 55Q91 · 55N91

\section{Introduction}

A decade ago, the authors [5] studied Real cobordism $M \mathbb{R}$, an $R O(\mathbb{Z} / 2)$-graded $\mathbb{Z} / 2$-equivariant spectrum discovered by Landweber [9], which is related to complex cobordism $M U$ in the same way as Atiyah's Real $K$-theory $K R$ [1] does to $K$-theory.

Communicated by Jim Stasheff.

P. Hu was supported in part by NSF Grant DMS 1104348. I. Kriz was supported by NSF Grant DMS 1102614.

P. Hu

Department of Mathematics, Wayne State University, Detroit, MI 48202, USA

e-mail: po@math.wayne.edu

I. Kriz $(\bowtie)$

Department of Mathematics, University of Michigan, Ann Arbor, MI 48109-1109, USA

e-mail: ikriz@umich.edu 
In particular, the authors computed the $R O(\mathbb{Z} / 2)$-graded coefficients of $M \mathbb{R}$. A key step in that computation was the fact that $M \mathbb{R}$ is a complete $\mathbb{Z} / 2$-equivariant spectrum, which means that the spectrum of its fixed points is equivalent to its spectrum of homotopy fixed points. At that time, the authors thought about the possibility of enhancing, in some way, the $\mathbb{Z} / 2$-action on $M \mathbb{R}$ or some modification of it and computing the coefficients in hopes of obtaining more homotopy-theoretical information, but didn't make much progress. The subject was recently revived in a spectacular way by Hill et al. [4] who solved negatively the famous Kervaire invariant 1 problem using an $R O(\mathbb{Z} / 8)$-graded $\mathbb{Z} / 8$-spectrum obtained, roughly speaking, from smashing four copies of $M \mathbb{R}$ and considering the $\mathbb{Z} / 8$-action which combines a cyclic permutation of the factors with the Real action.

In the present paper, we consider a more straightforward action on $M \mathbb{R}$ itself, by the group $\mathbb{Z} / 2 \times \mathbb{Z} / 2$. This action, also, gives rise to an $R O(\mathbb{Z} / 2 \times \mathbb{Z} / 2)$-graded spectrum (or, in the terminology of [10], spectrum indexed over the complete universe), which we call $M \mathbb{R}_{\mathbb{Z} / 2}$. The action is defined precisely in the next section, but roughly speaking, it combines the Real structure with a structure of " $\mathbb{Z} / 2$-equivariant cobordism". In fact, the authors were aware of this action while writing [5], but couldn't calculate the coefficients at that time.

Recently, the authors' interest in $M \mathbb{R}_{\mathbb{Z} / 2}$ was renewed for a reason unrelated to [4]: the construction of $M \mathbb{R}_{\mathbb{Z} / 2}$ is a direct cobordism analogue of Karoubi's topological

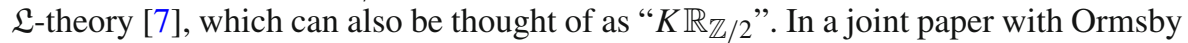
[6], the authors gave a set of foundations of $G$-equivariant stable motivic homotopy theory, and used it to give a solution to the homotopy fixed point problem for Karoubi's algebraic Hermitian $\mathrm{KH}$-theory for a field of characteristic 0 , where the full force of motivic homotopy theory can be brought to bear. [6] also contains a definition of algebraic Hermitian cobordism $M G L \mathbb{R}$. Algebraic Hermitian cobordism is still quite mysterious, but when we specialize to the field $\mathbb{R}$, similarly as in [7] for the case of $K$-theory, there is a topological version, which turns out to coincide with $M \mathbb{R}_{\mathbb{Z} / 2}$. Therefore, knowing more about $M \mathbb{R}_{\mathbb{Z} / 2}$ gives information about $M G L \mathbb{R}$.

At the same time, we also realized that $M \mathbb{R}_{\mathbb{Z} / 2}$ may be calculationally accessible, and this is the subject of the present paper. Interestingly, the techniques are a little different than we expected. In [4], a "slice spectral sequence" plays a crucial role. This is also the case in [6]. However, in the case of [6], the main point was to define a motivic analogue of the "Tate diagram" of Greenlees and May [3], and then use a slice spectral sequence to investigate Tate cohomology, which is more accessible than the Borel cohomology itself.

In the case of the $\mathbb{Z} / 2 \times \mathbb{Z} / 2$-spectrum $M \mathbb{R}_{\mathbb{Z} / 2}$, one also has an analogue of such "Tate diagram", but two things should be pointed out. First of all, the relevant diagram is not the case for $G=\mathbb{Z} / 2 \times \mathbb{Z} / 2$ of the Tate diagram canonically associated to $G$-equivariant spectra for any compact Lie group by Greenlees and May. The diagram we need is a generalized construction which brings more fully to bear the theory of universal spaces of families by Lewis et al. [10], to take a "Tate diagram" with respect to a $\mathbb{Z} / 2$ subgroup of $\mathbb{Z} / 2 \times \mathbb{Z} / 2$ (which we call $\mathbb{Z} / 2\{h\}$ ), and then consider separately the fixed points of the $\mathbb{Z} / 2\{h\}$-fixed point spectrum under the quotient $\mathbb{Z} / 2$. The other thing to realize is that $M \mathbb{R}_{\mathbb{Z} / 2}$ is not a complete spectrum, and thus our "Tate diagram" plays a somewhat different role than in completion theorems. The Tate diagram was 
previously used in a similar way by the second author in the simpler problem of computing the coefficients of $M U_{\mathbb{Z} / p}$ [8].

In the final step, we encounter $M \mathbb{R}^{*}$ cohomology groups of ( $\mathbb{Z} / 2$-fixed) stunted projective spaces, which is also a calculation we were aware of in [5] as desirable, and couldn't do. In the present paper, we do this calculation by taking advantage of the fact that $M \mathbb{R}$, again, is a complete spectrum. We completely identify the differentials of the corresponding Borel cohomology spectral sequence, and as a result compute an associated graded object of the $R O(\mathbb{Z} / 2 \times \mathbb{Z} / 2)$-graded coefficients of $M \mathbb{R}_{\mathbb{Z} / 2}$ with respect to a suitable complete filtration (Theorem 5); we lack a good enough "nomenclature" for the elements to solve all the extensions at this point. However, for a subring graded by certain special dimensions, we do have a complete answer as a ring (Theorem 3 ).

The present paper is organized as follows. All of our precise statements are too technical to make in the introduction. In the Sect. 2, we establish the notation to state the non-calculational part of our result. We also do all the relevant equivariant stable homotopy theory. In Sect. 3, we compute the $M \mathbb{R}^{*}$ cohomology of stunted projective spaces. In the Appendix, we say a few words on how $M \mathbb{R}_{\mathbb{Z} / 2}$ relates to $M G L \mathbb{R}$.

\section{The topological hermitian cobordism spectrum and its coefficients}

Notation: We begin with establishing some notation. $\mathbb{Z} / 2$-equivariant Real cobordism is a $\mathbb{Z} / 2 \times \mathbb{Z} / 2$-equivariant spectrum indexed over the complete universe. This means that we must distinguish carefully between the different elements of $\mathbb{Z} / 2 \times \mathbb{Z} / 2$ and its different real irreducible representations. We will denote the non-zero elements of $\mathbb{Z} / 2 \times \mathbb{Z} / 2$ by $g_{\alpha}, g_{\gamma}$ and $h$, and its non-trivial real irreducible representations by $\alpha, \gamma$ and $\gamma \alpha$ : By definition, on $\alpha, h, g_{\alpha}$ act by minus, on $\gamma, h, g_{\gamma}$ act by minus, on $\gamma \alpha, g_{\alpha}, g_{\gamma}$ act by minus. We will think of $g_{\gamma}, g_{\alpha}$ as "real structures", and of $h$ as the “Z/2-equivariant structure".

Accordingly, we will consider a complete complex universe, i.e.

$$
U=(\mathbb{C}[\mathbb{Z} / 2\{h\}])^{\infty} .
$$

We denote complex conjugation by $g_{\alpha}$, and put $g_{\gamma}=h g_{\alpha}$. (However, note that we can think of $g_{\gamma}$ as the complex conjugation.) For a $\mathbb{Z} / 2$-equivariant complex $\mathbb{Z} / 2$ representation $V$, denote by $\operatorname{Gr}(V, n)$ the space of all $n$-dimensional complex vector subspaces of $V$. Then as usual, there is a "tautological" $\mathbb{Z} / 2$-equivariant complex vector bundle $\gamma^{n}$ on $\operatorname{Gr}(V, n)$ where the fiber over an $n$-subspace $W \subset V$ is $W$. We let

$$
\left(M \mathbb{R}_{\mathbb{Z} / 2}\right)_{V}:=G r(U \oplus V,|V|)^{\gamma_{|V|}}
$$

(where the superscript denotes the Thom space). For $V \subset W \subset \subset U, G r(U \oplus V,|V|)$ is canonically embedded into $\operatorname{Gr}(U \oplus W,|W|)$ by adding $W-V$ (in the second summand), and accordingly the restriction of the bundle $\gamma_{|W|}$ on $\operatorname{Gr}(U \oplus W,|W|)$ to $G r(U \oplus V,|V|)$ canonically splits off the equivariant "trivial" (i.e. induced from a point) bundle $W-V$, i.e. we get a canonical map

$$
S^{W-V} \wedge\left(M \mathbb{R}_{\mathbb{Z} / 2}\right)_{V} \rightarrow\left(M \mathbb{R}_{\mathbb{Z} / 2}\right)_{W}
$$


Taking into account the whole $\mathbb{Z} / 2 \times \mathbb{Z} / 2$-action, the resulting $\mathbb{Z} / 2 \times \mathbb{Z} / 2$-equivariant spectrum indexed over the complete universe $U$ is what we denote by $M \mathbb{R}_{\mathbb{Z} / 2}$. It is worth commenting that the construction we described is obviously promoted to a $\mathbb{Z} / 2 \times \mathbb{Z} / 2$-equivariant symmetric spectrum, and hence $M \mathbb{R}_{\mathbb{Z} / 2}$ is a $\mathbb{Z} / 2 \times \mathbb{Z} / 2$ equivariant $E_{\infty}$ ring spectrum indexed over the complete universe (see [6]).

The goal of this paper is to compute the "coefficients"

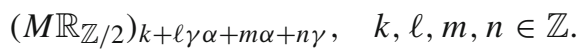

There is one simplification which we may deduce right away. Recall [2] that a $\mathbb{Z} / 2$-equivariant commutative associative ring spectrum $E$ indexed over the complete universe is called complex-oriented if for every $\mathbb{Z} / 2$-equivariant finite-dimensional complex vector bundle $\xi$ of dimension $n$ on a $\mathbb{Z} / 2$-equivariant $\mathrm{CW}$-complex $X$, there exists a Thom class, i.e. a class

$$
u_{\xi} \in \widetilde{E}^{2 n} X^{\xi}
$$

such that if we denote by $\theta: X^{\xi} \rightarrow X^{\xi} \wedge X_{+}$the Thom diagonal, then we have an isomorphism

$$
\theta^{*}\left(u_{\xi} \otimes ?\right): E^{k+\ell \alpha} X \stackrel{\cong}{\longrightarrow} \widetilde{E}^{k+\ell \alpha+2 n} X^{\xi}
$$

where $\alpha$ is the sign representation. (The definition really works for $G$-equivariant spectra for any finite abelian group $G$, but the case of $G=\mathbb{Z} / 2$ is the only one we need here.)

Observation 1 When $E$ is a complex-oriented $\mathbb{Z} / 2$-equivariant spectrum, then the coefficients of $E$ are $2-2 \alpha$-periodic. $M U_{\mathbb{Z} / 2}$ is a complex-oriented $\mathbb{Z} / 2$-equivariant spectrum.

Proof For the first statement, consider the $\mathbb{Z} / 2$-equivariant complex bundle $2 \alpha$ over a point. For the second statement, apply classification of equivariant complex $n$-bundles to $\operatorname{Gr}(U \oplus n, n)$.

Now following Atiyah [1], a $\mathbb{Z} / 2$-equivariant Real bundle is a $\mathbb{Z} / 2$-equivariant complex bundle with a $\mathbb{Z} / 2$-equivariant antilinear involution. Following the conventions at the beginning of this section, we call a $\mathbb{Z} / 2 \times \mathbb{Z} / 2$-equivariant commutative associative ring spectrum indexed over the complete universe Real-oriented if for every $n$-dimensional Real bundle on $\mathbb{Z} / 2 \times \mathbb{Z} / 2$-space $X$, there exists a Thom class, i.e. a class

$$
u_{\xi} \in \widetilde{E}^{n(1+\gamma \alpha)} X^{\xi}
$$

such that we have an isomorphism

$$
\theta^{*}\left(u_{\xi} \otimes ?\right): E^{k+\ell \alpha+m \gamma+r \gamma \alpha} X \stackrel{\cong}{\longrightarrow} \widetilde{E}^{k+\ell \alpha+m \gamma+r \gamma \alpha+n(1+\gamma \alpha)} X^{\xi} .
$$

Lemma 2 When $E$ is a Real-oriented $\mathbb{Z} / 2 \times \mathbb{Z} / 2$-equivariant spectrum, indexed over the complete universe, the coefficients of $E$ are $(\gamma+\alpha-1-\gamma \alpha)$-periodic. The spectrum $M \mathbb{R}_{\mathbb{Z} / 2}$ is Real-oriented. Additionally, we have 


$$
M \mathbb{R}_{k+\ell \alpha+m \gamma+n \gamma \alpha} \cong M \mathbb{R}_{k+m \alpha+\ell \gamma+n \gamma \alpha}
$$

Proof For the first statement, consider the Real $\mathbb{Z} / 2$-equivariant bundle $\alpha+\gamma$ over a point. For the second statement, consider the $n$-dimensional Real bundle classifying spaces $\operatorname{Gr}(U \oplus n, n)$. For the last statement, note that $\alpha$ and $\gamma$ play symmetric roles in the definition of $M \mathbb{R}_{\mathbb{Z} / 2}$.

Lemma 2 identifies certain dimensions in the coefficients of $M \mathbb{R}_{\mathbb{Z} / 2}$, so it reduces the set of separate "dimensions" we must consider. To get an further, however, we must substantially use equivariant stable homotopy theory, as developed in Lewis et al. [10]. The method we present here may well be more general, and useful in computing the coefficients of $G$-equivariant spectra where $G$ is a non-cyclic finite abelian group.

The strategy, in our case, is to investigate the $\mathbb{Z} / 2$-equivariant spectrum

$$
\left(M \mathbb{R}_{\mathbb{Z} / 2}\right)^{\mathbb{Z} / 2\{h\}} .
$$

This, in turn, can be computed by considering the " $\mathbb{Z} / 2\{h\}$-Tate diagram" in the language of Greenlees and May [3]. In our case, it is important to note that this is not the same approach as considering directly the $\mathbb{Z} / 2 \times \mathbb{Z} / 2$-equivariant Tate diagram. One must note that after taking $\mathbb{Z} / 2\{h\}$-fixed points, our "Tate diagram" retains a $\mathbb{Z} / 2$-equivariant action by taking

$$
\mathbb{Z} / 2=(\mathbb{Z} / 2 \times \mathbb{Z} / 2) /(\mathbb{Z} / 2\{h\}) .
$$

Fortunately, the foundations of what we need have been completely set up in [10]. Using the terminology of [10], we have a $\mathbb{Z} / 2 \times \mathbb{Z} / 2$-equivariant cofibration sequence

$$
E \mathcal{F}_{+} \rightarrow S^{0} \rightarrow S^{\infty \alpha+\infty \gamma}
$$

where $\mathcal{F}$ is the family of subgroups disjoint with $\mathbb{Z} / 2\{h\}$ (recall that the universal space of a family $\mathcal{F}$ of subgroups of a finite group $G$ is a $G-C W$ complex $E \mathcal{F}$ such that for $H \subseteq G, E \mathcal{F}^{H}$ is contractible when $H \in \mathcal{F}$ and empty otherwise). The diagram we have in mind is

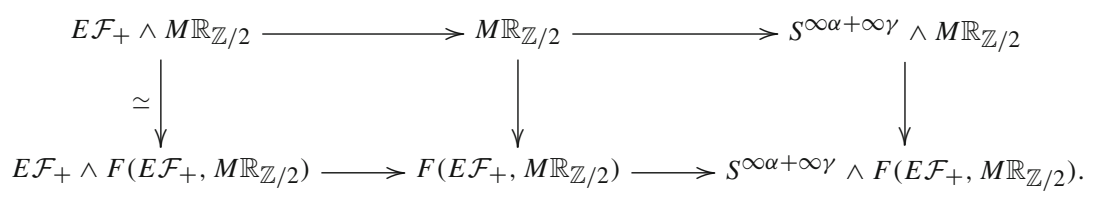

The reason the left column is an equivalence is as follows: For a family $\mathcal{F}$ of subgroups of $G$, we have a notion of $\mathcal{F}$-equivalence of $G$-equivariant spectra, which means equivalence on $H$-fixed points for every $H \in \mathcal{F}$. Now it is obvious that the natural map

$$
M \mathbb{R}_{\mathbb{Z} / 2} \rightarrow F\left(E \mathbb{Z} / 2_{+}, M \mathbb{R}_{\mathbb{Z} / 2}\right)
$$


is an $\mathcal{F}$-equivalence, and hence by Lemma 2.12 of [10], the left hand column of (6) is an $\mathcal{F}$-equivalence. However, these spectra are homotopy equivalent to $\mathcal{F}$-CW spectra (i.e. CW-spectra whose cells are of the form $G / H_{+} \wedge D^{n}, n \in \mathbb{Z}, H \in \mathcal{F}$ ), and by Theorem 2.2 of [10], the left hand column of (6) is a weak equivalence). Now applying $(?)^{\mathbb{Z} / 2\{h\}}$ to (6), we therefore obtain a (weak) homotopy pullback

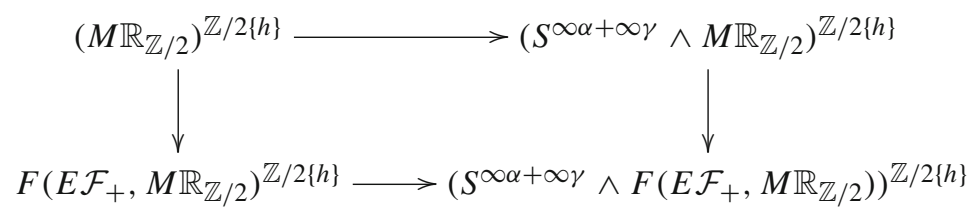

It is also worth noting that by the Adams isomorphism [10] Theorem 7.1, the homotopy fiber of the rows of (7) is

$$
\left(E \mathcal{F}_{+} \wedge M \mathbb{R}_{\mathbb{Z} / 2}\right) /(\mathbb{Z} / 2\{h\})
$$

Now the main point of our method is that the upper left, lower left and lower right corners of the pullback (7) can be calculated directly. In many was, in fact, the computation is analogous to [8]; if we do not do any suspensions by sums of copies of $\alpha$ or $\gamma$, the complications introduced by the Real structure are in fact only minor. We will treat this case first, in part because in this case, we have a more precise theorem.

Regarding the upper right corner, the main idea is that we can compute the $\mathbb{Z} / 2\{h\}$ fixed point of the spectrum $S^{\infty \alpha+\infty \gamma} \wedge M \mathbb{R}_{\mathbb{Z} / 2}$ on the prespectrum level and then take the colimit. This relies on a result of Lewis et al. [10] that in general, for a normal subgroup $H$ of a (say) finite group $G$, and a based CW $G$-space $X$, if we denote by $\mathcal{F}_{H}$ the family of subgroups not containing $H$, and consider the cofibration sequence

$$
\left(E \mathcal{F}_{H}\right)_{+} \rightarrow S^{0} \rightarrow \widetilde{E \mathcal{F}_{H}}
$$

then we have an equivalence of $G / H$-spectra indexed over the complete universe

$$
\left(\widetilde{E \mathcal{F}_{H}} \wedge \Sigma_{G}^{\infty} X\right)^{H} \simeq \Sigma_{H}^{\infty} X^{H}
$$

Note that in the case discussed in this paper, $\mathcal{F}_{\mathbb{Z} / 2\{h\}}$ coincides with the family of subgroups of $\mathbb{Z} / 2 \times \mathbb{Z} / 2$ disjoint with $\mathbb{Z} / 2\{h\}$. Similarly as in tom Dieck [11] (cf. [8]), this then identifies the upper right corner as

$$
\bigvee_{n \in \mathbb{Z}} \Sigma^{n(1+\gamma \alpha)} B \mathbb{U}_{+} \wedge M \mathbb{R}
$$

where $\mathbb{U}$ is the infinite unitary group with $\mathbb{Z} / 2$-action by complex conjugation.

The lower left corner of (7) is computed as follows: Consider the inclusion of universes 


$$
i: U^{\mathbb{Z} / 2\{h\}} \rightarrow U
$$

Then over $U^{\mathbb{Z} / 2\{h\}}$, we have a “ $\mathbb{Z} / 2\{h\}$-fixed Real cobordism spectrum with completely replicates the definition of $M \mathbb{R}_{\mathbb{Z} / 2}$ with $U$ replaced by $U^{\mathbb{Z} / 2\{h\}}$ (so the action of $\mathbb{Z} / 2\{h\}$ on all spaces constituting the prespectrum is trivial). This is also essentially the same construction as the construction of $M \mathbb{R}$ (cf. [5]), so we will also denote this $U^{\mathbb{Z} / 2\{h\}}$. indexed $\mathbb{Z} / 2 \times \mathbb{Z} / 2$-spectrum by $M \mathbb{R}$. Then we have a standard map

$$
M \mathbb{R} \rightarrow i^{*} M \mathbb{R}_{\mathbb{Z} / 2}
$$

which is an $\mathcal{F}$-equivalence, so the natural map on $\mathbb{Z} / 2\{h\}$-fixed points is a $\mathbb{Z} / 2$ equivariant weak equivalence:

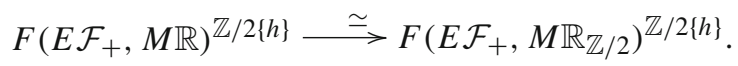

(Recall that in applying $(?)^{\mathbb{Z} / 2\{h\}}$ to a spectrum indexed over the complete universe, we apply $i^{*}$ implicitly first.)

The left hand side of (11) is, by definition,

$$
F\left(E \mathcal{F} /(\mathbb{Z} / 2\{h\})_{+}, M \mathbb{R}\right) .
$$

However, we have

$$
E \mathcal{F} /(\mathbb{Z} / 2\{h\}) \simeq B_{\mathbb{Z} / 2}(\mathbb{Z} / 2)
$$

(the right hand side is, by definition, the classifying space of $\mathbb{Z} / 2$-equivariant principal $\mathbb{Z} / 2$-bundles). In the next section, we will show that the natural inclusion

$$
B_{\mathbb{Z} / 2}(\mathbb{Z} / 2) \rightarrow B \mathbb{S}^{1}
$$

(where $\mathbb{S}^{1}$ denotes the unit sphere in $\mathbb{C}$ with $\mathbb{Z} / 2$-action by complex conjugation) induces in $M \mathbb{R}$-cohomology (in dimensions $k+\ell \gamma \alpha, k, \ell \in \mathbb{Z}$ ) the map

$$
M \mathbb{R}^{*} B \mathbb{S}^{1}=M \mathbb{R}^{*}[[u]] \rightarrow M \mathbb{R}^{*}[[u]] /[2]_{F}(u)
$$

where $F$ is the universal formal group law, and $u$ is a variable in dimension $-1-\gamma \alpha$ (just as in [5], in an effort to prevent constant confusions, all gradings are homological).

Combining (9), (11), (13), we get that the diagram of the coefficients of the upper right, lower left and lower right corner of the diagram (7) in dimensions $k+\ell \gamma \alpha, k, \ell \in$ $\mathbb{Z}$ is

$$
\begin{gathered}
M \mathbb{R}^{*}\left[u, u^{-1}\right]\left[b_{1}, b_{2}, \ldots\right] \\
\qquad \mathbb{R}^{*}[[u]] /[2]_{F}(u) \longrightarrow\left(M \mathbb{R}^{*}[[u]] /[2]_{F}(u)\right)\left[u^{-1}\right]
\end{gathered}
$$


The generators $b_{k}$ are in dimensions $k(1+\gamma \alpha)$ and just as in [8], Theorem 1.1, the images of $b_{k}$ are the coefficients of $x^{k}$ in $x+{ }_{F} u$. Also, just as in [8], the images of the upper right and lower left corners span the lower right corner, and we get

Theorem 3 The bigraded module of coefficients

$$
\left(\left(M \mathbb{R}_{\mathbb{Z} / 2}\right)_{k+\ell \gamma \alpha}\right)_{k, \ell \in \mathbb{Z}}
$$

is isomorphic to the pullback of the diagram of rings (14).

Of course, we are not done with computing the $R O(\mathbb{Z} / 2 \times \mathbb{Z} / 2)$-graded coefficients of $M \mathbb{R}_{\mathbb{Z} / 2}$, since we must also discuss suspensions by linear combinations, with coefficients in $\mathbb{Z}$, of $\alpha$ and $\gamma$. Let us realize first precisely which coefficients are left to compute. We claim, in fact, that it suffices to compute

$$
\left(M \mathbb{R}_{\mathbb{Z} / 2}\right)_{k+\ell \gamma \alpha+m \alpha}, \quad m<0 .
$$

To see this, consider an arbitrary coefficient (2). By Lemma 2, we may assume, say, that $m \leq n$. Using the periodicity in Lemma 2, however, we may then add $n$ to $k, \ell$ and subtract $n$ from $m, n$ and the resulting isomorphic coefficient is either of the form (15) (when $m<n$ ), or of the form covered in Theorem 3 (when $m=n$ ).

Now the idea is to consider (15) one $m$ at a time. This means replacing the diagram (7) by

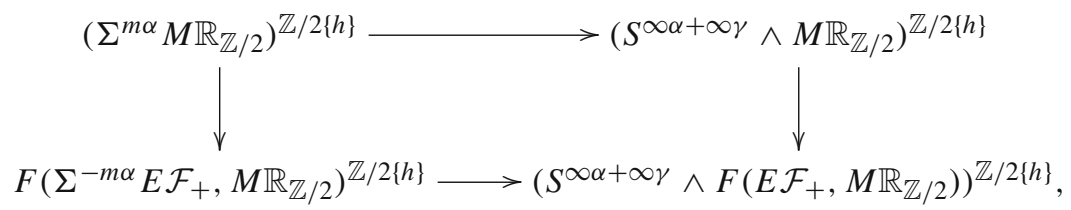

$-m>0$. (We realize that the right column is periodic with respect to suspending by a multiple of $\alpha$.) Therefore, the main task remaining is to compute

$$
F\left(\Sigma^{-m \alpha} E \mathcal{F}_{+}, M \mathbb{R}_{\mathbb{Z} / 2}\right)^{\mathbb{Z} / 2\{h\}} .
$$

Using the "splitting" (10), we get that (17) is equivalent to

$$
F\left(\Sigma^{-m \alpha} E \mathcal{F}_{+}, M \mathbb{R}\right)^{\mathbb{Z} / 2\{h\}} \simeq F\left(\left(\Sigma^{-m \alpha} E \mathcal{F}_{+}\right) /(\mathbb{Z} / 2\{h\}), M \mathbb{R}_{\mathbb{Z} / 2}\right) .
$$

Note that the right hand side is simply a $\mathbb{Z} / 2$-spectrum. But we can do better. Recall (12). It follows that

$$
\left(\Sigma^{-m \alpha} E \mathcal{F}_{+}\right) /(\mathbb{Z} / 2\{h\}) \simeq\left(B_{\mathbb{Z} / 2}(\mathbb{Z} / 2)\right)^{-m \gamma_{1}}
$$

where $\gamma^{1}$ is the "tautological" 1 -dimensional real bundle on $B_{\mathbb{Z} / 2}(\mathbb{Z} / 2)$, induced by the sign representation of $\mathbb{Z} / 2$ on $\mathbb{R}$. Additionally, again, if we consider the fixed $\mathbb{Z} / 2$-space $\mathbb{R} P^{\infty}$, we have a canonical inclusion 


$$
\left(\mathbb{R} P^{\infty}\right)^{-m \gamma_{1}} \rightarrow\left(B_{\mathbb{Z} / 2}(\mathbb{Z} / 2)\right)^{-m \gamma_{1}},
$$

which, since $M \mathbb{R}$ is a complete spectrum, induces an equivalence

$$
F\left(\left(B_{\mathbb{Z} / 2}(\mathbb{Z} / 2)\right)^{-m \gamma_{1}}, M \mathbb{R}\right) \simeq F\left(\left(\mathbb{R} P^{\infty}\right)^{-m \gamma_{1}}, M \mathbb{R}\right)
$$

Note that one commonly refers to $\left(\mathbb{R} P^{\infty}\right)^{-m \gamma_{1}}$ as the stunted projective space $\mathbb{R} P_{-m}^{\infty}$, so our problem is reduced to computing

$$
\widetilde{M \mathbb{R}^{*}}\left(\mathbb{R} P_{-m}^{\infty}\right), \quad-m \geq 0
$$

(where $\mathbb{R} P_{-m}^{\infty}$ is considered as a fixed $\mathbb{Z} / 2$-space), and the bottom row of diagram (16), which, in view of naturality and Theorem 3, follows from knowing

$$
\widetilde{M \mathbb{R}^{*}}\left(\mathbb{R} P_{-m}^{\infty}\right) \rightarrow M \mathbb{R}^{*}\left(\mathbb{R} P^{\infty}\right),
$$

induced by the "0-section" map

$$
\mathbb{R} P^{\infty} \rightarrow \mathbb{R} P_{-m}^{\infty}, \quad m>0 .
$$

Unfortunately, we cannot determine (22) completely. The main problem is that we have no obvious "pretty" expression for (21) akin to (13). What we do have is a complete computation of the Borel cohomology spectral sequence (BCSS) for (22), and the map to the BCSS for $M \mathbb{R}^{*} \mathbb{R} P^{\infty}$. Ordinarily, one would think this is not sufficient information to determine the coefficients of the upper left corner of (7) or even its associated graded object, since taking associated graded objects does not preserve pullbacks. However, in the present case, the pullbacks we are dealing with are rather special, and therefore it is possible to state a theorem on this level of precision. Let us begin with a preliminary lemma.

Let $F^{i}, i \geq 0$ be a decreasing filtration on an abelian group $X$. We will call this filtration complete if the natural map

$$
X \rightarrow \lim X / F^{i} X
$$

is an isomorphism.

Lemma 4 Suppose we have a cartesian co-cartesian square of abelian groups

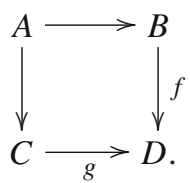

(Recall that this is the same thing as a cartesian square, or pullback diagram, such that $\operatorname{Im}(f)+\operatorname{Im}(g)=D$.) Suppose further we have complete decreasing filtrations $F^{i}, i \geq 0$ on $B, C, D$ such that 


$$
f\left(F^{i} B\right)+g\left(F^{i} C\right)=F^{i} D .
$$

Then forming the pullback

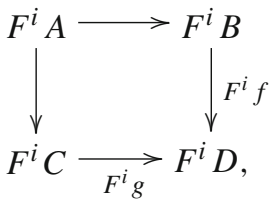

$\left(F^{i} A\right)_{i}$ is a complete decreasing filtration on $A$, and we also have a pullback of the corresponding associated graded objects

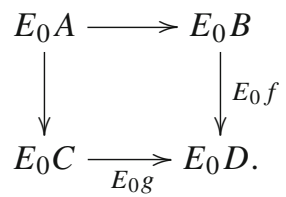

Proof We may view as a cartesian co-cartesian square (24) as a short exact sequence

$$
0 \longrightarrow A \longrightarrow B \oplus C \stackrel{f \oplus g}{\longrightarrow} D \longrightarrow 0 .
$$

Assuming (26), we have a short exact sequence embedded into (28)

$$
0 \longrightarrow F^{i} A \longrightarrow F^{i} B \oplus F^{i} C \stackrel{F^{i} f \oplus F^{i} g}{\longrightarrow} F^{i} D \longrightarrow 0 .
$$

Therefore, we have a quotient short exact sequence

$$
0 \longrightarrow A / F^{i} A \longrightarrow B / F^{i} B \oplus C / F^{i} C \stackrel{f / F^{i} f \oplus g / F^{i} g}{\longrightarrow} D / F^{i} D \longrightarrow 0 \text {. }
$$

Now interpreting (30) as a pullback again, taking inverse limits over $i$ and using the commutation of categorical limits gives the completeness of the filtration $\left(F^{i} A\right)_{i}$. Taking the quotient of (29) by the same short exact sequence with $i$ replaced by $i+1$ gives the last statement.

Consider now the fixed point inclusion of $\mathbb{Z} / 2 \times \mathbb{Z} / 2$-spaces

$$
b: S^{0} \rightarrow S^{\gamma \alpha}
$$

Theorem 5 There is a complete filtration on (15) such that the associated graded object is isomorphic to the pullback of the diagram 


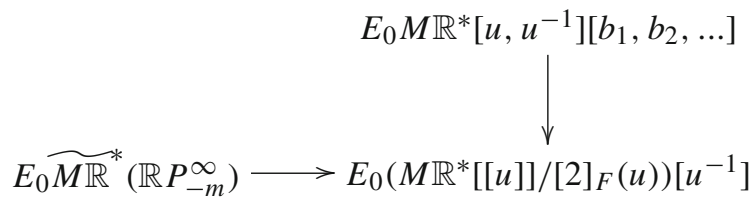

where in the upper right, lower left and lower right corner of (32), E $E_{0}$ denotes the associated graded object of the decreasing filtration by powers of the ideal $(b)$. The lower horizontal arrow of (32) is as computed in Theorems 11, 12 below.

Comment: It is not being asserted that the filtration of (2) mentioned in the Theorem is the filtration by powers of the ideal $(b)$.

Proof (with the exception of the last statement, which is proved in the next section). The strategy is to show that the $R O(\mathbb{Z} / 2)$-graded coefficients of diagram (16) satisfy the hypotheses of Lemma 4 . To this end, note that the lower left corner is $M \mathbb{R}^{*}$ cohomology of a spectrum (reduced cohomology of a space, actually), so the filtration by powers of $(b)$ is simply the BCSS filtration, and the statement follows from the convergence of the corresponding BCSS (to be completely precise, the BCSS is only conditionally convergent, so the proof follows from our complete computation of the differentials, which is done in the next section).

To prove completeness of the filtration by powers of $(b)$ in the lower right and upper right corner of the diagram (32), our strategy is to show that any infinite series of elements in the same dimension divisible by an increasing power of $b$ can be written as an infinite series of elements divisible by an increasing power of $b$ using only $u^{m}, m \geq-n$ with constant $n$. Such series then converge in $u^{-n} M \mathbb{R}^{*}[[u]] /[2]_{F}(u)$, which is a shift of $M \mathbb{R}^{*} \mathbb{R} P^{\infty}$, where the series converges by the completeness of $M \mathbb{R}$ (since the filtration by powers of $(b)$ is the filtration associated with the BCSS).

The statement asserted in the last paragraph follows, in effect, from dimensional considerations. Recall from [5] (or verify directly) that the cokernel of the canonical inclusion

$$
\mathbb{Z}[a] /(2 a) \rightarrow M \mathbb{R}_{k+\ell \alpha}
$$

is 0 when $k+\ell<0$. Since $u^{-1}$ is of dimension $1+\alpha$ (from the point of view of $M \mathbb{R}$, the dimension is $1+\gamma \alpha$ from the point of view of our entire calculation), if we have a homogeneous infinite series as mentioned in the last paragraph involving powers of $u$ not bounded below, then the $M \mathbb{R}_{*}$-coefficients of those powers must be in the image of (33). (In the case of the upper right corner, recall that $b_{k}$ is in dimension $k(1+\alpha)$.) One easily sees however that as we increase the power of $a$ in (33), adding no multiple of $1+\alpha$ can bring the elements into the same dimension. This proves the required completeness statement.

To complete verifying the assumptions of Lemma 4, it remains to verify (25). This is done as follows: Denote by $\gamma_{1}^{\mathbb{C}}$ the $\mathbb{Z} / 2$-equivariant Real line bundle on $B_{\mathbb{Z} / 2}(\mathbb{Z} / 2)$ given by letting the generator of $\mathbb{Z} / 2$ act on $\mathbb{C}$ by -1 . Then we have the " 0 -section map" 


$$
\iota:\left(B_{\mathbb{Z} / 2}(\mathbb{Z} / 2)\right)^{\gamma_{1}} \rightarrow\left(B_{\mathbb{Z} / 2}(\mathbb{Z} / 2)\right)^{\gamma_{1}^{\mathbb{C}}}
$$

Applying $M \mathbb{R}^{*}$, and inverting $u$, we get a diagram

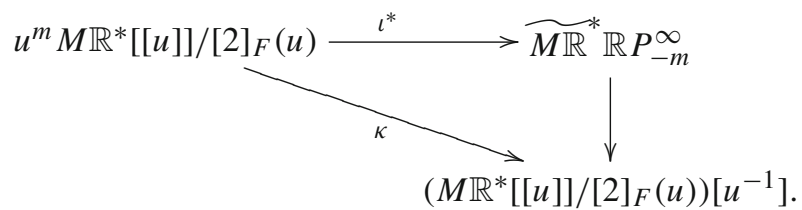

In the upper right corner, we use the equivalence (20) again, and in the upper left corner we use the Real orientation of $-m \gamma_{1}^{\mathbb{C}}$. In fact, this also implies that $\kappa$ is the ordinary localization map, so its image together with the image of the vertical map (14) span $\left(M \mathbb{R}^{*}[[u]] /[2]_{F}(u)\right)\left[u^{-1}\right]$. This implies (25) for $i=0$. Note, however, that since $F^{i}=b^{i} F^{0}$ in all the three terms of (32), the general case follows.

\section{The Real cobordism of stunted projective spaces}

The following result is well known, but we restate it to make the exposition selfcontained:

Lemma 6 Let $E_{* *}^{*}$ be a spectral sequence (graded homologically). Suppose we have numbers $p, s$ and a morphism of spectral sequences

$$
\phi: E_{* *}^{\prime *} \rightarrow E_{* *}^{*}
$$

which is an isomorphism on $E_{m, q}^{s}$-terms for $m \leq p$, and $E_{m, q}^{s}=0$ for $m>p$. Then for $r \geq s, m \leq p$

$$
E_{*, *}^{\prime r} \cong E_{*, *}^{r} \oplus \underset{s \leq i<r}{\bigoplus} \operatorname{Im}\left(d_{c}^{i}\right)
$$

where $d_{c}^{i}$ is the restriction of $d^{i}$ to

$$
E_{>p, *}^{i} \rightarrow E_{\leq p, *}^{i}
$$

Furthermore, in the isomorphism (36), $\phi$ corresponds to the projection to the first summand. Similarly, suppose we have a morphism of spectral sequences

$$
\psi: E_{* *}^{*} \rightarrow E_{/ * *}^{*}
$$

which is an isomorphism on $E_{m, q}^{s}$-terms for $m>p$, and $E_{m, q}^{s}=0$ for $m \leq p$. Then for $r \geq s, m \geq p$

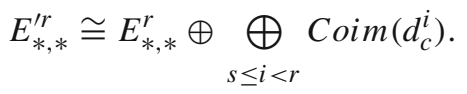


Furthermore, in the isomorphism (37), $\psi$ corresponds to the injection of the first summand.

Proof Induction on $r$. For $r=s$, the statement is assumed. Consider now, say, $\phi$. Assuming the induction hypothesis for a given $r$, the second summand by definition may be non-zero only in filtration degrees $>p-r+1$, and hence $\phi$ is an isomorphism on $E_{\leq p-r+1, *}^{r}$-terms. This means that $E_{\leq p-r, *}^{\prime r}$, which can be taken as the codomain of $d^{r}$-differentials in $E^{\prime r}$, is mapped by $\phi$ monorphically, and hence $\phi d_{r}=d_{r} \phi$ determines $d_{r}$. This implies the statement with $r$ replaced by $r+1$, hence completing the induction. The proof of the statement for $\psi$ is analogous.

For example, this lemma (applied with $s=1$ ) implies that quite generally, the differentials of a Borel homology and Borel cohomology spectral sequence with respect to a cyclic group are precisely the restrictions of the differentials in the corresponding Tate spectral sequence (see also [3]).

Let $\mathbb{R} P^{\infty}$ denote the infinite real projective space with trivial $\mathbb{Z} / 2$-action. observe that

$$
\mathbb{R} P^{\infty}=\left(B \mathbb{S}^{1}\right)^{\mathbb{Z} / 2}
$$

where $\mathbb{S}^{1}$ is the unit sphere in $\mathbb{C}$ with the $\mathbb{Z} / 2$-action of complex conjugation. Recall [5] that by the theory of Real-oriented spectra,

$$
B P \mathbb{R}^{*} B \mathbb{S}^{1}=B P \mathbb{R}^{*}[[u]],
$$

where the dimension of $u$, graded homologically, is $-1-\alpha$.

Proposition 7 The relation (38) induces an isomorphism

$$
B P \mathbb{R}^{*} \mathbb{R} P^{\infty} \cong B P \mathbb{R}^{*}[[u]] /[2]_{F}(u) .
$$

Proof If we denote by $\mathbb{S}^{\infty}$ the unit sphere in $\mathbb{C}^{\infty}$ with $\mathbb{Z} / 2$-action by complex conjugation, then $\mathbb{S}^{\infty} /(\mathbb{Z} / 2)$ is the unit sphere of the Real line bundle $\left(\gamma_{1}\right)^{2}$ on $B \mathbb{S}^{1}$ where $\gamma^{1}$ is the tautological bundle. Thus, we have a cofibration sequence

$$
\mathbb{S}^{\infty} /(\mathbb{Z} / 2) \rightarrow B \mathbb{S}^{1} \rightarrow\left(B \mathbb{S}^{1}\right)^{\left(\gamma^{1}\right)^{2}}
$$

which gives an isomorphism

$$
B P \mathbb{R}^{*}\left(\mathbb{S}^{\infty} /(\mathbb{Z} / 2)\right) \cong B P \mathbb{R}^{*}[[u]] /[2]_{F}(u) .
$$

On the other hand, notice that (38) factors through the natural equivariant embedding

$$
\mathbb{R} P^{\infty}=\left(\mathbb{S}^{\infty}\right)^{\mathbb{Z} / 2} /(\mathbb{Z} / 2) \subset \mathbb{S}^{\infty} /(\mathbb{Z} / 2)
$$

which is an equivalence non-equivariantly and hence induces an isomorphism in $B P \mathbb{R}$ cohomology, since $B P \mathbb{R}$ is a complete spectrum. This completes the proof. 
Note that (39) is not an equivalence equivariantly: in fact, the right hand side is $B_{\mathbb{Z} / 2}(\mathbb{Z} / 2)$, the classifying space for $\mathbb{Z} / 2$-equivariant $\mathbb{Z} / 2$-principal bundles.

Even though Proposition 7 gives a complete calculation of $B P \mathbb{R}^{*}\left(\mathbb{R} P^{\infty}\right)$, for the purposes of comparison with stunted projective spaces, we need to study its Borel cohomology spectral sequence. Let us begin by studying the $[2]_{F}$-series in $B P \mathbb{R}_{*}$ further. Even though one may think this is the same as the [2] ${ }_{F}$-series in $B P_{*}$, there are some fundamental differences. For example, the natural map

$$
B P^{*}[[u]] /\left(\frac{[2]_{F}(u)}{u}\right) \rightarrow B P^{*}[[u]] /\left(\frac{[2]_{F}(u)}{u}\right)\left[u^{-1}\right]
$$

is an inclusion. This is not the case when we replace $B P^{*}$ by $B P \mathbb{R}^{*}$. If we choose a natural number $n$, then in $B P \mathbb{R}^{*}[[u]]$,

$$
a^{2^{n}-1}\left([2]_{F}(u)+v_{n} u^{2^{n}}\right) \equiv a^{2^{n}-1}\left(v_{n} u^{2^{n}}+v_{n+1} u^{2^{n+1}}\right) \quad \bmod u^{2^{n+1}+1} .
$$

We denote the left hand side by $a^{2^{n}-1} \phi_{n} u^{2^{n}}$.

We shall also put

$$
w_{n} a^{2^{n}-1} u^{2^{n}}:=a^{2^{n}-1}\left(\phi_{n}-v_{n}\right) .
$$

Here we treat $a^{2^{n}-1} \phi_{n} u^{2^{n}}, w_{n} a^{2^{n}-1} u^{2^{n}}$. By abuse of notation, we use the same symbols also for their representative in a Borel cohomology spectral sequence. We do not assign a meaning to $w_{n}, \phi_{n}$ at this point. However, as is common in calculating spectral sequences, we will need to discuss "corresponding" elements in different spectral sequences. The suggestive notation allows more flexibility in this direction. For example, the representative of $a^{2^{n}-1} \phi_{n} u^{2^{n}}$ in a Borel cohomology spectral sequence can be multiplied by powers of $a$. Also, in related spectral sequences, additional elements may sometimes survive, allowing for the notation $\phi_{n}, w_{n}$ to "spread". (For example, in a Tate cohomology spectral sequence, $a$ becomes invertible, so we can write $\phi_{n} u^{2^{n}}$.

Those types of liberties with notation may seem dangerous, and in general they are. We are, however, safe in the special type of argument we are using, which is to identify enough differentials in the Tate cohomology spectral sequence to get the right $E_{\infty}$-term, and then conclude that none other can occur. We will see the differentials from [5], and in the case of stunted projective spaces, also other differentials which come from comparisons of spectral sequences. The final "jigsaw puzzle" fitting the differentials together is quite complicated. In this context, insisting on a separate name of elements in each spectral sequence (or worse yet, each page) would become terribly cumbersome.

Lemma 8 The Borel cohomology spectral sequence for $B P \mathbb{R}^{*}\left(\mathbb{R} P^{\infty}\right)$ has $E^{1}$-term

$$
B P^{*}[a]\left[\sigma, \sigma^{-1}\right][[u]] /[2]_{F}(u)
$$


where, to conform with [5], everything is graded homologically, $\left|v_{n}\right|=\left(2^{n}-1\right)(1+$ $\alpha),|a|=-\alpha,|\sigma|=\alpha-1,|u|=-1-\alpha$. The differentials are

$$
d\left(x \sigma^{-2^{n}}\right)=x a^{2^{n+1}-1} v_{n},
$$

where

$$
x \in \mathbb{Z} / 2\left[v_{n}, v_{n+1}, \ldots\right][a]\left[\sigma^{ \pm 2^{n+1}}\right] u^{j}
$$

with

$$
0 \leq j<2^{n}
$$

(when $n=0, \mathbb{Z} / 2\left[v_{0}, v_{1} \ldots\right]$ is replaced by $B P_{*}$ ) and

$$
d\left(y \sigma^{-2^{n}}\right)=y a^{2^{n+1}-1} w_{n},
$$

where

$$
y \in \mathbb{Z} / 2\left[v_{n+1}, \ldots\right][a]\left[\sigma^{ \pm 2^{n+1}}\right] u^{j}
$$

with

$$
j \geq 2^{n}
$$

Proof The statement about the $E^{1}$-term is obvious. The fact that $u$ is a permanent cycle follows from Proposition 7. The differential (41) follows from the fact that the Borel cohomology spectral sequence (BCSS) for $B P \mathbb{R}^{*} \mathbb{R} P^{\infty}$ is a module over the BCSS for $B P \mathbb{R}^{*}$. The differential (43) is a rewriting or (41) by the discussion in the paragraph preceding the Lemma. (The point is that for $j \geq 2^{n}$, the leading term is already wiped out by the differential (41), but the same differential still has a non-zero target by the congruence (40).) There can be no further differentials by Lemma 6 since, as one easily checks, after introducing the differentials (41), (43), the corresponding "Tate spectral sequence" (i.e. the spectral sequence obtained by inverting $a$ ) converges to its correct target, $H \mathbb{Z} / 2^{*} \mathbb{R} P^{\infty}\left[a, a^{-1}\right]$.

Remark It is important to realize that Tate cohomology is the generalized cohomology theory represented by its spectrum in the Tate diagram. The Tate cohomology spectral sequence converges to generalized Tate cohomology. For a space, such as $\mathbb{R} P^{\infty}$, which is not a finite cell complex, the generalized Tate cohomology is not obtained by inverting $a$ in the Borel cohomology. Thereby, the 2 -series $[2]_{F}(u)$ is 0 in $\widetilde{B P \mathbb{R}}^{*}\left(\mathbb{R} P^{\infty}\right)$, but not in $\left(B P \mathbb{R}^{*}\left(\mathbb{R} P^{\infty}\right)\right)\left[a^{-1}\right]$.

We shall not tackle the case of stunted projective spaces. In this case, additional differentials do occur.

Lemma 9 When $q=2 p$, and

$$
\ell=2^{n_{1}}-2^{n_{2}}+\cdots-2^{n_{q}},
$$


$n_{1}>\cdots>n_{q}>0$ or $q=2 p+1$ and

$$
\ell=2^{n_{1}}-2^{n_{2}}+\cdots+2^{n_{q}}-1,
$$

$n_{1}>\cdots>n_{q}>0$, then in the BCSS for $B P \mathbb{R}^{*} \mathbb{R} P_{2 \ell+1}^{\infty}$,

$$
d u^{\ell+1}=\sigma^{2^{n} q} v_{q} a^{2^{n q+1}-1} u^{\ell+1} .
$$

Proof As an induction hypothesis, we claim that the kernel of the Tate cohomology spectral sequence (TCSS) map induced by the projection

$$
\mathbb{R} P^{\infty} \rightarrow \mathbb{R} P_{2 \ell+1}^{\infty}
$$

where $\ell \equiv 2^{k} \bmod 2^{k+1}$ or $\ell \equiv 2^{k}+1 \bmod 2^{k+1}$ on the $2^{m}-1$ 'st page, $m \leq k$, is a free $\mathbb{Z} / 2\left[v_{m}, v_{m+1}, \ldots\right]\left[a, a^{-1}\right]\left[\sigma^{ \pm 2^{m}}\right]$-module $M_{m}$ on the generators

$$
\phi_{m} u^{\ell+i}, \quad 1 \leq i \leq 2^{m} \text {. }
$$

For $m=1$, this is a statement about the $E^{1}$-term. Suppose it is true for a given $m$. Then first of all, $d^{<2^{m+1}-1}$ of $\sigma^{-2^{n}} u^{\ell+1}$ must be contained in $M_{m}$ (because the differential is 0 in the TCSS associated to $B P \mathbb{R}^{*} \mathbb{R} P^{\infty}$ ). However, we see that $d^{1}$ is excluded by explicit formula (this is also related to the different behavior of $\ell$ even and odd). For dimensional reasons, $d^{>1}$ can only arise from a shift of power of $\sigma$, so $d^{2^{m+1}-1}$ is the lowest possible differential (corresponding to shift by $\sigma^{2^{m}}$ ), so $d^{<2^{m+1}-1}$ cannot occur.

Now from considering the map of TCSS associated with

$$
\Sigma^{2 \ell+1} M \mathbb{Z} / 2 \rightarrow \mathbb{R} P_{2 \ell+1}^{\infty},
$$

we see that for $m<\ell$,

$$
d^{2^{m+1}-1} \sigma^{-2^{m}} u^{\ell+1}=v_{m} a^{2^{m+1}-1} u^{\ell+1}+\sum_{1<i \leq 2^{m}} x_{i} u^{\ell+i} a^{2^{m+1}-1}
$$

where $x_{i} \in \phi_{m} \mathbb{Z} / 2\left[v_{n}, v_{n+1}, \ldots\right]$ (the power of $\sigma$ is excluded for dimensional reasons). But then by the module structure over the TCSS for $B P \mathbb{R}^{*}$,

$$
d^{2^{m+1}-1} u^{\ell+1}=\sum_{1<i \leq 2^{m}} \sigma^{2^{m}} x_{i} u^{\ell+i} a^{2^{m+1}-1} .
$$

However, for the lowest $i$ for which $x_{i} \neq 0$, we then have by (50)

$$
d^{2^{m+1}-1}\left(\sigma^{2^{m}} x_{i} u^{\ell+i} a^{2^{m+1}-1}\right) \neq 0 \quad \bmod u^{\ell+i+1},
$$


which is a contradiction with $d^{2^{m+1}-1}$ being a differential. Therefore, $x_{i}=0$ for all $i$ and

$$
d^{2^{m+1}-1} \sigma^{-2^{m}} u^{\ell+1}=v_{m} a^{2^{m+1}-1} u^{\ell+1}
$$

and

$$
d^{2^{m+1}-1} \phi_{m} u^{\ell+1}=0 .
$$

This, in turn, implies the induction step.

Now let us consider $d^{2^{k+1}-1}$. Then by (49),

$$
\begin{gathered}
d^{2^{\ell+1}-1} u^{\ell+1}=\phi_{\ell} \sigma^{2^{\ell}} a^{2^{\ell+1}-1}+\sum_{1<i \leq 2^{\ell}} x_{i} u^{\ell+i} a^{2^{m+1}-1}, \\
d^{2^{\ell+1}-1}\left(u^{\ell+1} \sigma^{-2^{\ell}}\right)=w_{\ell} a^{2^{\ell+1}-1}+\sum_{1<i \leq 2^{\ell}} x_{i} u^{\ell+i} \sigma^{-2^{\ell}} a^{2^{\ell+1}-1} .
\end{gathered}
$$

Again, however, for the lowest $i$ for which $x_{i} \neq 0$, we conclude that then

$$
d^{2^{\ell+1}-1}\left(x_{i} u^{\ell+i} \sigma^{-2^{\ell}} a^{2^{\ell+1}-1}\right) \neq 0 \quad \bmod u^{\ell+i+1},
$$

which is a contradiction with $d^{2^{\ell+1}-1}$ being a differential. This implies that $x_{i}=0$ for all $i$, and the statement of the Lemma.

Now let $\ell$ be as in (45) or (46). Let

$$
\epsilon(\ell):=2 \ell-2^{n_{1}}+1 \text {. }
$$

Let further

$$
\begin{aligned}
\ell_{1} & :=\ell, \\
\ell_{i+1} & :=2^{n_{i}}-\ell_{i}-1, i<q .
\end{aligned}
$$

Set

$$
\epsilon_{i}:=1+\ell+\epsilon\left(\ell_{q}\right)+\cdots+\epsilon\left(\ell_{i+1}\right) .
$$

Lemma 10 For $0 \leq i \leq q$, in the BCSS for $B P \mathbb{R}^{*} \mathbb{R} P_{2 \ell+1}^{\infty}$ we have

$$
d\left(u^{\epsilon_{i}}\right)=\sigma^{2^{n_{i}}} v_{n_{i}} a^{2^{n_{i}+1}-1} u^{\epsilon_{i}} .
$$


Proof One notes that if one puts

$$
\ell^{\prime}:=2^{n_{1}}-2^{n_{2}}+\cdots-2^{n_{i}} \text { if } i \text { is even }
$$

and

$$
\ell^{\prime}:=2^{n_{1}}-\cdots+2^{n_{i}}+1 \text { if } i \text { is odd, }
$$

then

$$
\ell^{\prime} \leq \epsilon\left(\ell_{q}\right)+\cdots+\epsilon\left(\ell_{i+1}\right)<\ell^{\prime}+2^{n_{i}}-1 .
$$

If $i$ is odd, then $\ell^{\prime} \geq \ell$, so we have a map

$$
\mathbb{R} P_{2 \ell+1}^{\infty} \rightarrow \mathbb{R} P_{2 \ell^{\prime}+1}^{\infty},
$$

and we may therefore deduce the differential from Lemma 9 applied to $\ell^{\prime}$ in place of $\ell$.

When $i$ is even, then $\ell^{\prime} \leq \ell$, so we have a map in the opposite direction

$$
\mathbb{R} P_{2 \ell^{\prime}+1}^{\infty} \rightarrow \mathbb{R} P_{2 \ell+1}^{\infty},
$$

so the TCSS for $B P \mathbb{R}^{*} \mathbb{R} P_{2 \ell^{\prime}+1}^{\infty}$ detects the differential, but a priori, we don't know that there isn't an error term. However, in this case, we may proceed by reversed induction on $i$ : if the statement is correct for $i^{\prime}>i$, then we may use the differentials already proved and the module structure of the TCSS $E^{\prime}$ corresponding to $B P \mathbb{R}^{*} \mathbb{R} P_{2 \ell^{\prime}+1}^{\infty}$, and the TCSS $E$ corresponding to $B P \mathbb{R}^{*} \mathbb{R} P_{2 \ell+1}^{\infty}$, and prove that the map

$$
E^{2^{n_{i}+1}-1} \rightarrow E^{2^{n_{i}+1}-1}
$$

is injective, and hence the differential in the target determines the differential in the source (see the statement of Theorem 11 which spells out this calculation explicitly).

Theorem 11 The BCSS for $B P \mathbb{R}^{*} \mathbb{R} P_{2 k+1}^{\infty}$ has $E^{1}$-term

$$
u^{k+1} B P_{*}[a]\left[\sigma, \sigma^{-1}\right][[u]] /\left(\frac{[2]_{F}(u)}{u}\right),
$$

$(|u|=-1-\alpha)$. The differentials are as follows: For each $i \geq k+1$, there are numbers $s_{k, i} \leq t_{k, i} \in\{1,2, \ldots, \infty\}$ such that we have (41) with (42) for

$$
0 \leq p<s_{k, i}
$$

or

$$
p=t_{k, i},
$$


and (43) with (44) for

$$
s_{k, i} \leq p<t_{k, i}
$$

Additionally, we have the differential

$$
d z=v_{p} a^{2^{p+1}-1} \sigma^{2^{p}}, \quad p=t_{k, i}
$$

with

$$
z \in u^{i} \mathbb{Z} / 2\left[v_{p}, v_{p+1}, \ldots\right][a]\left[\sigma^{ \pm 2^{p+1}}\right] .
$$

These are the only differentials in this BCSS.

The numbers $s_{k, i}, t_{k, i}$ are determined as follows: For $k=2^{\ell}-1$, put

$$
\begin{aligned}
t_{k, i}=\ell & \text { for } k+1 \leq i \leq 2 k, \\
t_{k, i}=\infty & \text { for } i>2 k .
\end{aligned}
$$

We put

$$
s_{k, i}=j \text { for } k+2^{j-1} \leq i<k+2^{j}, 1 \leq j \leq \ell,
$$

and

$$
s_{k, i}=j \text { for } i>2 k, 2^{j} \leq i<2^{j+1} .
$$

For $k$ not of the form $2^{\ell}-1$, let $k+1 \leq 2^{\ell} \leq 2 k$. Then

$$
\begin{aligned}
& s_{k, i}=s_{2^{\ell}-k-1,2^{\ell}-2 k-1+i} \\
& t_{k, i}=t_{2^{\ell}-k-1,2^{\ell}-2 k-1+i}
\end{aligned} \text { for } i<2^{\ell},
$$

and if we let

$$
2^{\ell}-k \leq 2^{m}<2^{\ell+1}-2 k-2,
$$

then for $2^{\ell} \leq i \leq 2 k$, put

$$
t_{k, i}=\ell, s_{k, i}=j
$$

for

$$
m<j \leq \ell, 2 k+1-2^{\ell}+2^{j-1} \leq i<2 k+1-2^{\ell}+2^{j} .
$$

For $i>2 k$, put

$$
t_{k, i}=\infty, s_{k, i}=j
$$

for $2^{j} \leq i<2^{j+1}$ 
Proof The differentials (51) with (52) follows from Lemma 10. The remaining differentials follow from the module structure over the BCSS for $B P \mathbb{R}^{*} \mathbb{R} P^{\infty}$. Considering the corresponding TCSS, one observes that these differentials get the correct answer for

$$
\widetilde{B P \mathbb{R}^{*}} \mathbb{R} P_{2 k+1}^{\infty}=H \mathbb{Z} / 2^{*} \mathbb{R} P_{2 k+1}^{\infty},
$$

and hence no other differentials can occur.

The result for $B P \mathbb{R}^{*} \mathbb{R} P_{2 k}^{\infty}$ is analogous, although the pattern is a little different.

Theorem 12 The BCSS for $B P \mathbb{R} * \mathbb{R} P_{2 k}^{\infty}$ has $E^{1}$-term

$$
u^{k} B P_{*}[a]\left[\sigma, \sigma^{-1}\right][[u]] /\left([2]_{F}(u)\right),
$$

$(|u|=-1-\alpha)$. The differentials are as follows: For each $i \geq k$, there are numbers $a_{k, i} \leq b_{k, i} \in\{0,2, \ldots, \infty\}$ such that we have (41) with (42), (43) with (44) and (51) with (52) occur as in Theorem 11 with $s_{k, i}$ replaced by $a_{k, i}$ and $t_{k, i}$ replaced by $b_{k, i}$.

The numbers $a_{k, i}, b_{k, i}$ are determined as follows: For $k=2^{\ell}$, put

$$
\begin{aligned}
b_{k, i}=\ell & \text { for } k \leq i<2 k, \\
b_{k, i}=\infty & \text { for } i \geq 2 k .
\end{aligned}
$$

We put

$$
a_{k, i}=j \quad \text { for } i=k \text { and } 0=j, \text { or } k+2^{j-1} \leq i<k+2^{j}, 1 \leq j \leq \ell,
$$

and

$$
a_{k, i}=j \quad \text { for } i \geq 2 k, 2^{j} \leq i<2^{j+1} .
$$

For $k$ not of the form $2^{\ell}$, let $k \leq 2^{\ell}<2 k$. Then

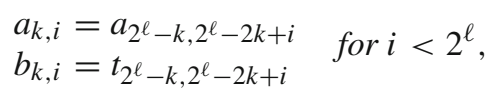

and if we let

$$
2^{\ell}-k \leq 2^{m}<2^{\ell+1}-2 k,
$$

then for $2^{\ell} \leq i<2 k$, put

$$
b_{k, i}=\ell, a_{k, i}=j
$$

for

$$
m<j \leq \ell, 2 k-2^{\ell}+2^{j-1} \leq i<2 k-2^{\ell}+2^{j} .
$$


For $i>2 k$, put

$$
b_{k, i}=\infty, a_{k, i}=j
$$

for $2^{j} \leq i<2^{j+1}$.

The proof is completely analogous to the proof of Theorem 11, so we only record the main steps. Lemma 9 is replaced by

\section{Lemma 13 When}

$$
\ell=2^{n_{1}}-2^{n_{2}}+\cdots \pm 2^{n_{q}},
$$

(the sign is + resp. - when $q$ is odd resp. even), $n_{1}>\cdots>n_{q-1}>n_{q}+1$, then in the BCSS for $B P \mathbb{R}^{*} \mathbb{R} P_{2 \ell}^{\infty}$, there is a differential

$$
d u^{\ell}=u^{\ell} \sigma^{2^{n q}} v_{n_{q}} a^{2^{n} q}-1 .
$$

Proof Analogous to the proof of Lemma 9. The principal difference is that (49) is replaced by the inclusion of the bottom cell

$$
S^{2 \ell} \rightarrow \mathbb{R} P_{2 \ell}^{\infty} .
$$

To get the analogue of Lemma 10 , let $\ell$ be as in (53), and let

$$
\delta(\ell):=2 \ell=2^{n_{1}},
$$

and let

$$
\begin{aligned}
\ell_{1}: & =\ell, \\
\ell_{i+1} & :=2^{n_{i}}-\ell_{i}, i<q .
\end{aligned}
$$

Set

$$
\delta_{i}:=\ell+\delta\left(\ell_{i+1}\right)+\cdots+\delta\left(\ell_{q}\right) .
$$

The analogue of Lemma 10 then reads

Lemma 14 For $0 \leq i \leq q$,

$$
d\left(u^{\delta_{i}}\right)=\sigma^{2^{n_{i}}} v_{n_{i}} a^{2^{n_{i}+1}-1} u^{\delta_{i}} .
$$

The proof is analogous to the proof of Lemma 10, and the proof of Theorem 12 is analogous to the proof of Theorem 11. 
Example Let us compute the Borel cohomology spectral sequence for $B P \mathbb{R}^{*} \mathbb{R} P_{101}^{\infty}$. This is the case of Theorem 11 with $k=50$. For the purposes of the theorem, we write

$$
k=2^{6}-2^{4}+2^{2}-2^{1} .
$$

We refer to the differentials (41) as vertical, the differentials (43) as horizontal and the differentials (51) as special.

In this terminology, we get special non-zero differentials

$$
\begin{aligned}
& d_{3} \sigma^{0} \bmod { }^{4} u^{51}, \\
& d_{7} \sigma^{0} \bmod { }^{8} u^{52}, \\
& d_{31} \sigma^{0} \bmod 32 u^{i}, \quad 53 \leq i \leq 63, \\
& d_{127} \sigma^{0} \bmod 128 u^{i}, \quad 64 \leq i \leq 100 .
\end{aligned}
$$

In addition, we have vertical differentials

$$
\begin{aligned}
& d_{15} \sigma^{8} \bmod 16, \quad 53 \leq i \leq 56, \\
& d_{63} \sigma^{32} \bmod 64 u^{i}, \quad 64 \leq i \leq 68
\end{aligned}
$$

and horizontal differentials

$$
\begin{aligned}
& d_{1} \sigma^{1} \bmod 2, \quad i \geq 51, \\
& d_{3} \sigma^{2} \bmod 4, \quad i \geq 51, \\
& d_{7} \sigma^{4} \bmod 8 u^{i}, \quad i \geq 52 \\
& d_{15} \sigma^{8} \bmod 16, \quad i \geq 56, \\
& d_{31} \sigma^{16} \bmod 32, \quad i \geq 53 \\
& d_{63} \sigma^{32} \bmod 64, \quad i \geq 69 \\
& d_{127} \sigma^{64} \bmod 128, \quad i \geq 64 .
\end{aligned}
$$

Differentials $d \sigma^{j} u^{i}$ for $i \geq 101$ are the same as in the BCSS for $B P \mathbb{R}^{*} \mathbb{R} P^{\infty}$.

Example Let us compute the Borel cohomology spectral sequence for $B P \mathbb{R}^{*} \mathbb{R} P_{100}^{\infty}$. This is the case of Theorem 12 with $k=50$. For the purposes of the theorem, we write

$$
k=2^{6}-2^{4}+2^{1}
$$

In the above terminology, we get special differentials

$$
\begin{aligned}
& d_{3} \sigma^{0} \quad \bmod 4 u^{i}, \quad i=50,51, \\
& d_{31} \sigma^{0} \bmod 32 u^{i}, \quad 52 \leq i \leq 63, \\
& d_{127} \sigma^{0} \bmod 128 u^{i}, \quad 64 \leq i \leq 99 .
\end{aligned}
$$


In addition, we have vertical differentials

$$
\begin{aligned}
& d_{1} \sigma^{1} \bmod 2 u^{50}, \\
& d_{15} \sigma^{8} \bmod 16, \quad 52 \leq i \leq 55, \\
& d_{63} \sigma^{32} \bmod 64 u^{i}, \quad 64 \leq i \leq 67
\end{aligned}
$$

and horizontal differentials

$$
\begin{aligned}
& d_{1} \sigma^{1} \bmod 2, \quad i \geq 51, \\
& d_{3} \sigma^{2} \bmod 4, \quad i \geq 50, \\
& d_{7} \sigma^{4} \bmod 8 u^{i}, \quad i \geq 52, \\
& d_{15} \sigma^{8} \bmod 16, \quad i \geq 56, \\
& d_{31} \sigma^{16} \bmod 32, \quad i \geq 52, \\
& d_{63} \sigma^{32} \bmod 64, \quad i \geq 68, \\
& d_{127} \sigma^{64} \bmod 128, \quad i \geq 64 .
\end{aligned}
$$

Differentials $d \sigma^{j} u^{i}$ for $i \geq 100$ are the same as in the BCSS for $B P \mathbb{R}^{*} \mathbb{R} P^{\infty}$.

\section{Appendix: The topological realization of algebraic hermitian cobordism}

Let us start with a warm-up case, Karoubi's topological Hermitian $K$-theory [7] $\mathfrak{L}$, which is the topological realization of his algebraic Hermitian $K$-theory over the field $\mathbb{R}$. When considered as a $\mathbb{Z} / 2 \times \mathbb{Z} / 2$-equivariant spectrum indexed over the complete universe, this is represented as follows: One chooses a complex universe $U_{B} \cong \mathbb{C}^{\infty}$ together with a Real structure ? and a hyperbolic Real form $B$. Then the $\mathfrak{L}^{0}$ is represented by the $\mathbb{Z} / 2 \times \mathbb{Z} / 2$-equivariant space $B G L\left(U_{B}\right)$, the classifying space of the group $G L\left(U_{B}\right)$ which is the direct limit of groups of the form $G L\left(V_{B}\right)$ where $V_{B}$ are finite-dimensional Real vector spaces endowed with the $\mathbb{Z} / 2 \times \mathbb{Z} / 2$-action where one generator acts by complex conjugation, and the other by

$$
A \mapsto\left(A^{T}\right)^{-1}
$$

where (?) $)^{T}$ denotes the adjoint matrix with respect to the symmetric bilinear form $B$ :

$$
B(A x, y)=B\left(x, A^{T} y\right) .
$$

The link with $\mathbb{Z} / 2$-equivariant Real $K$-theory is obtained as follows. Since we assumed we have a Real structure on $V_{B}$, we have a unique splitting

$$
V_{B}=\left(V_{B}\right)^{+} \oplus\left(V_{B}\right)^{-}
$$

such that $\left(V_{B}\right)^{+},\left(V_{B}\right)^{-}$are $B$-orthogonal, and $B$ is positive definite on $\left(V_{B}\right)^{+}$and negative definite on $\left(V_{B}\right)^{-}$. Then define a positive definite symmetric bilinear form 
$B_{+}$to be equal to $B$ on $\left(V_{B}\right)^{+}$and to $-B$ on $\left(V_{B}\right)^{-}$. Now consider the Hermitian form on $V_{B}$ defined by

$$
\langle u, v\rangle=B_{+}(u, \bar{V}) .
$$

Let $U\left(V_{B}\right)$ be the group of unitary matrices with respect to this Hermitian form. Then we have a map

$$
U\left(V_{B}\right) \rightarrow G L\left(V_{B}\right)
$$

which is easily seen to be a weak $\mathbb{Z} / 2 \times \mathbb{Z} / 2$-equivalence. Moreover, define a $\mathbb{Z} / 2$ action on $V_{B}$ such that the generator $h$ acts by 1 on $\left(V_{B}\right)^{+}$and by -1 on $\left(V_{B}\right)^{-}$. The key point then is that restricted to $U\left(V_{B}\right),(56)$ is complex conjugation, conjugated by $h$. Passing to direct limit, this gives an equivalence of the 0 -space of $\mathbb{Z} / 2$-equivariant Real $K$-theory to $\mathfrak{L}$. One checks that the periodicity maps given by Karoubi [7] coincide,

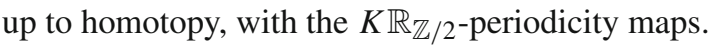

To treat topological Hermitian cobordism, which is the $\mathbb{Z} / 2 \times \mathbb{Z} / 2$-equivariant spectrum indexed over the complete universe which is the topological realization of the $\mathbb{Z} / 2$-equivariant motivic spectrum $M G L \mathbb{R}$ over the field $\mathbb{R}$, we must enrich (58) to "Thom spaces". To this end, recall [6] that the "Thom space" in that case is obtained as the homotopy cofiber of the map from the "unit sphere bundle" to the base space. The reason of the quotation marks is that the we cannot take the complement of the 0 -section in the vector bundle, since this would not have a $\mathbb{Z} / 2$-action. Instead, the "unit sphere" is emulated by the quadric

$$
B(x, y)=1, \quad x, y \in V_{B}
$$

which has both a $\left.G L_{(} V_{B}\right)$ action where $A$ acts by $A$ on the $x$-coordinates and by $\left(A^{T}\right)^{-1}$ on the $y$ coordinates. Note that projection to the $x$-coordinates (or the $y$-coordinates) is a motivic homotopy equivalence onto $V_{B}-\{0\}$.

For the purposes or comparison of the topological realization of this with $\mathbb{Z} / 2$ equivariant Real cobordism, we take the topological subspace $S_{+}$of (59) consisting of all points of (59) where

$$
y=\overline{h(x)} .
$$

By projecting to the $x$-coordinates, this is homeomorphic to the set of all points of $V_{B}$ satisfying

$$
B_{+}(x, \bar{x})=1,
$$

which is the unit sphere. Thus, the inclusion of $S_{+}$to the quadric (59) is a (nonequivariant homotopy equivalence). Now one sees, however, that the restriction of the $G L\left(V_{B}\right)$-action on (59) restricts to an action of $U\left(V_{B}\right)$ on $S^{+}$, and that, in effect, the $\mathbb{Z} / 2 \ltimes U\left(V_{B}\right)$-space $S_{+}$is isomorphic to the unit sphere of $V_{B}$ with respect to the positive-definite Hermitian form (57). On the other hand, the inclusion of $S^{+}$to (59) is a $\mathbb{Z} / 2 \ltimes U\left(V_{B}\right)$-homotopy equivalence. Thus, we have exhibited homotopy equivalences of the homotopy cofiber of the projection

$$
B\left(S_{+}, U\left(V_{B}\right), *\right)_{+} \rightarrow B U\left(V_{B}\right)_{+}
$$

both to the $V_{B}$-space of the topological realization of $M G L \mathbb{R}$ over $\mathbb{R}$ and of $M \mathbb{R}_{\mathbb{Z} / 2}$. One easily checks that the structure maps coincide as well. 


\section{References}

1. Atiyah, M.F.: K-theory and reality. Q. J. Math. Oxf. Sec. (2) 17, 367-386 (1966)

2. Cole, M., Greenlees, J.P.C., Kriz, I.: Equivariant formal group laws. Proc. Lond. Math. Soc. (3) 81(2), 355-386 (2000)

3. Greenlees, J.P.C., May, J.P.: Generalized Tate cohomology. Mem. Am. Math. Soc. 113(543), viii+178 pp (1995)

4. Hill, M.A., Hopkins, M.J., Ravenel, D.C.: On the non-existence of elements of Kervaire invariant 1. arXiv:0908.3724

5. Hu, P., Kriz, I.: Real-oriented homotopy theory and an analogue of the Adams-Novikov spectral sequence. Topology 40(2), 317-399 (2001)

6. Hu, P., Kriz, I., Ormsby, K.: The homotopy limit problem for Hermitian K-theory, equivariant motivic homotopy theory and motivic Real cobordism. Adv. Math. 228(1), 434-480 (2011)

7. Karoubi, M.: Periodicite de la $K$-Theorie Hermitienne. Lecture Notes in Mathematics, vol. 343, pp. 301-411. Springer, Berlin (1973)

8. Kriz, I.: The $\mathbb{Z} / p$-equivariant cobordism ring. Contemp. Math. 239, 217-223 (1999)

9. Landweber, P.S.: Conjugations on complex manifolds and equivariant homotopy of $M U$. Bull. Am. Math. Soc. 74, 271-274 (1968)

10. Lewis, L.G. Jr., May, J.P., Steinberger, M.: Equivariant Stable Homotopy Theory. With contributions by J.E. McClure. Lecture Notes in Mathematics, vol. 1213, x+538 pp. Springer, Berlin (1986)

11. Tom Dieck, T.: Bordism of $G$-manifolds and integrality theorems. Topology 9, 345-358 (1970) 